\title{
Platelet-rich fibrin exudate promotes the proliferation and osteogenic differentiation of human periodontal ligament cells in vitro
}

\author{
XIAOJU LI ${ }^{1 *}$, HUIXIAO YANG ${ }^{2 *}$, ZIJIIAN ZHANG ${ }^{3}$, ZHONGHAI YAN $^{3}$, HULING LV $^{2}$, YAN ZHANG ${ }^{4}$ and BIN WU ${ }^{1}$ \\ ${ }^{1}$ Department of Stomatology, The People's Hospital of Longhua, Shenzhen, Guangdong 518109; \\ ${ }^{2}$ Key Laboratory of Oral Medicine, Guangzhou Institute of Oral Disease, Stomatological Hospital of \\ Guangzhou Medical University, Guangzhou, Guangdong 510140, P.R. China; ${ }^{3}$ Department of Biomedical Sciences, \\ Paul L. Foster School of Medicine, Texas Tech University Health Sciences Center El Paso, El Paso, TX 79905, USA; \\ ${ }^{4}$ Department of General Therapy Dentistry, Stomatology Hospital of Jilin University, Changchun, Jilin 130021, P.R. China
}

Received February 23, 2018; Accepted August 13, 2018

DOI: $10.3892 / \mathrm{mmr} .2018 .9472$

\begin{abstract}
The purpose of the present study was to evaluate the effects of platelet-rich fibrin (PRF) exudate on the proliferation, osteogenic differentiation and mineralization of human periodontal ligament cells (hPDLCs) in vitro. In the present study PRF was obtained with permission, from the peripheral blood of healthy donors and PRF exudates were collected on the 7th day of incubation. hPDLCs were obtained from healthy premolars, cultured by a tissue explant method and identified with anti-vimentin and anti-cytokeratin antibodies. PRF exudates were added to hPDLCs in different concentrations to evaluate cell proliferation and osteogenic differentiation. The proliferation of hPDLCs was measured using a colorimetric assay. Osteogenic differentiation and mineralization were determined by Alizarin red staining, alkaline phosphatase activity (ALP), western blotting and reverse transcriptionquantitative polymerase chain reaction. Cell proliferation was enhanced by addition of the PRF exudate, which also promoted the formation of mineralized matrix nodules and upregulated ALP activity and osteoblast-associated levels of osteocalcin, runt-related transcription factor and osterix gene expression. As these stimulatory effects occurred in a dose-dependent manner, it was concluded that high concentrations of the PRF exudate served an essential role in the proliferation, osteogenic differentiation and mineralization of hPDLCs in vitro.
\end{abstract}

Correspondence to: Dr Huixiao Yang, Key Laboratory of Oral Medicine, Guangzhou Institute of Oral Disease, Stomatological Hospital of Guangzhou Medical University, 59 Huangsha Road, Guangzhou, Guangdong 510140, P.R. China

E-mail: yanghuixiao34@outlook.com

*Contributed equally

Key words: mineralization, osteogenic differentiation, periodontal ligament cells, platelet-rich fibrin, growth factor
The present study demonstrated that PRF exudate enhanced hPDLC proliferation, induced the osteoblastic differentiation of hPDLCs into mineralized tissue-formation cells in vitro, and may therefore provide potential benefits for periodontal tissue engineering; contributing to the primary processes of periodontal tissue regeneration. From the perspective of both economics and biology, PRF has greater clinical benefits than analogous growth factors.

\section{Introduction}

Periodontitis is a common inflammatory disease that results in irreversible destruction of periodontal tissues, including the periodontal ligament, alveolar bone and root cementum, and is the primary reason for tooth loss in adults. Currently, there are numerous challenges for treating periodontitis in clinical practice. A number of treatment approaches have been developed in the past decades and the canonical treatment of periodontal bone defects includes guided tissue regeneration (1), bone grafts (2), and application of enamel matrix derivative (3). However, these conventional therapies cannot be regarded as best-practice regenerative techniques due to their limited success and unpredictable prognosis.

Adult periodontal ligament stem cells (PDLSCs) were first discovered in 2004 (4). Since then, stem cell-based periodontal tissue regeneration treatment has attracted increasing attention in the field of dentistry. Furthermore, as an important cell type for periodontal tissue regeneration, PDLSCs have been reported to exhibit the ability to differentiate into osteoblasts and cementoblasts in vitro (5). However, the number and sources of autologous PDLSCs are limited, thereby hampering their clinical application. It is of great urgency to produce novel bioactive substances with the ability to enhance the proliferation, differentiation and osteogenic-associated gene/protein expression of PDLSCs.

In 2000, the French researcher Choukroun et al developed a straightforward open-access platelet-rich fibrin (PRF) protocol $(6,7)$. Choukroun's platelet-rich fibrin (PRF) has been considered as a second-generation platelet concentrate (6-8). 
When activated by thrombin and other triggers, platelets release a large number of growth factors, chemokines, and cytokines, which can interact with target protein receptors and induce cell proliferation, cell differentiation, and bone formation. As an autologous biomaterial, synthetic collagen PRF has been applied in a number of medical fields, including oral and maxillofacial surgery (9), plastic surgery, and dental implantology (10-13).

PRF is fibrin in a three-dimensional structure that contains a number of growth factors, including platelet-derived growth factor $\mathrm{AB}$ and transforming growth factor $\beta 1$ (TGF- $\beta 1$ ), and these are continuously released from the PRF matrix for $>7$ days (14). A number of studies have reported that PRF stimulates the proliferation and differentiation of a variety of cell types in vitro $(15,16)$. Studies have demonstrated that platelet-rich plasma can promote the proliferation of bone mesenchymal stem cells, fat source stem cells and skeletal muscle satellite cells as well as osteogenic differentiation (17). However, there is little recent evidence regarding the effects of PRF exudates on the proliferation and differentiation of human PDLCs (hPDLCs).

The objective of the present study was to assess the biological effects of different concentrations of PRF exudate on hPDLCs by measuring cell proliferation, alkaline phosphatase (ALP) activity, as well as osteocalcin $(O C N)$, runt-related transcription factor $1(R U N X 2)$ and osterix (OSX) gene expression.

\section{Materials and methods}

Preparation of hPRF exudates. The present study was approved by the Ethics Committee of the Jilin University Health Science Center (Jilin, China). In accordance with this committee, the hPRF exudate was prepared from three healthy male donors who had visited the outpatient clinic at the Jilin University Health Science Center between March 2017 and August 2017. They were nonsmokers and nondrinkers with age range 22-30 years, and gave their informed consent. Patient blood samples $(50 \mathrm{ml})$ were used to produce hPRF according to an existing protocol (8). Briefly, the samples were centrifuged at $750 \mathrm{x}$ g for $12 \mathrm{~min}$ at $10^{\circ} \mathrm{C}$. A white PRF clot formed between the acellular plasma and red blood cells (RBCs; Fig. 1A), and the clot was held by sterile forceps and separated from the RBCs using scissors (Fig. 1B). The clot was placed on the grid of an endo box and compressed by the endo box cover. Following $1 \mathrm{~min}$ of applied pressure, the PRF clot was converted into PRF membrane and the exudate was collected in the tray of the endo box (Fig. 1C).

The PRF exudate was centrifuged at $500 \mathrm{xg}$ for $5 \mathrm{~min}$ at $10^{\circ} \mathrm{C}$ to obtain exudate without RBCs, which were precipitated. The exudate was filtered using a $0.22 \mu \mathrm{m}$ sterile syringe filter unit (EMD Millipore, Billerica, MA, USA; cat. no. 01730). Pooled PRF exudates were stored at $-80^{\circ} \mathrm{C}$ prior to use. The original concentration of RF exudate was defined as $100 \%$ and various concentrations were obtained by dilution with minimal essential medium $\alpha$ ( $\alpha$-MEM; Gibco; Thermo Fisher Scientific, Inc., Waltham, MA, USA). A total of three PRF exudate concentrations were used in the present study: $100 \%$ (E1), 20\% (E2) and 4\% (E3).

hPDLC culture. A total of 10 healthy and noncarious premolars from 2 female and 3 male donors aged 13-18 years old, who had received orthodontic treatment at the Oral and Maxillofacial Surgery Department of the Stomatology School of Jilin University (Changchun, China) between March 2017 and August 2017, were obtained with informed consent. Periodontal ligaments were gently scraped from the middle third of the tooth-root surface with a sharp scalpel, minced with ophthalmic scissors and rinsed with $\alpha$-MEM (Gibco; Thermo Fisher Scientific, Inc.). These explants were cultured in $\alpha$-MEM supplemented with $15 \%$ fetal bovine serum (FBS; Thermo Fisher Scientific Inc.) and 1\% streptomycin and penicillin (Gibco; Thermo Fisher Scientific, Inc.), and the cultured pieces were then incubated at $37^{\circ} \mathrm{C}$ in an environment containing $5 \% \mathrm{CO}_{2}$. Examination by inverted light microscopy (Olympus Corporation, Tokyo, Japan) was carried out daily, and the medium was changed every three days. When the cell colony-formation units reached $80 \%$ confluence, the cells were transferred to a $75-\mathrm{cm}^{2}$ flask and this was defined as passage 1 . The same procedure was carried out repeatedly to produce multiple passages. Feeding was continued according to the cell-culturing protocol and only cells prior to passage 6 were used in the present study.

Immunocytochemistry staining. hPDLCs at passage 3 $\left(0.05 \times 10^{6}\right)$ were seeded into 24 -well plates and covered in advance with $14-\mathrm{mm}$ diameter circular coverslips and incubated for $48 \mathrm{~h}$. The cells were then rinsed 3 times with $0.01 \mathrm{M}$ phosphate-buffered saline (PBS) and then fixed with $4 \%$ paraformaldehyde for $20 \mathrm{~min}$ at room temperature. Following washing with PBS, $0.25 \%$ Triton X-100 was added to the 24 -well plates, which were incubated at $37^{\circ} \mathrm{C}$ for $15 \mathrm{~min}$. Endogenous peroxidase activity was eliminated by incubation with $3 \%$ $\mathrm{H}_{2} \mathrm{O}_{2}$ for $10 \mathrm{~min}$ at room temperature. Cells were then incubated with $1 \%$ bovine serum albumin (Gibco; Thermo Fisher Scientific, Inc.) and $22.52 \mathrm{mg} / \mathrm{ml}$ glycine in PBS + 0.1\% Tween 20 for $30 \mathrm{~min}$ at room temperature to block unspecific binding of antibodies. The cells were then incubated with anti-vimentin (1:100; cat. no. ab24525; Abcam, Cambridge, MA, USA) and anti-cytokeratin (1:200; cat. no. AM06387SU-N; OriGene Technologies, Inc., Beijing, China) primary antibodies overnight at $4^{\circ} \mathrm{C}$. Secondary goat-anti-rabbit, goat-anti-mouse and goat anti-chicken IgG were AlexaFluor 488 (cat. no. A-11008; Invitrogen; Thermo Fisher Scientific, Inc.), 568 (cat. no. A-11004; Invitrogen; Thermo Fisher Scientific, Inc.), and 647 (cat. no. A-21449; Invitrogen; Thermo Fisher Scientific, Inc.) labeled, respectively; and used in various combinations at a 1:1,000 dilution. The SP immunohistochemistry assay kit (OriGene Technologies, Inc.) was used for immunocytochemical staining according to the manufacturer's protocol and the diaminobenzidine kit (OriGene Technologies, Inc.) was used to stain positive cells. An inverted phase-contrast microscope (IX73; Olympus Corporation, Tokyo, Japan) was used to observe stained hPDLCs at magnifications of $\mathrm{x} 20$ or $\mathrm{x} 40$.

Proliferation analysis using the Cell Counting Kit-8 (CCK-8) assay. CCK-8 (Dojindo Molecular Technologies, Inc., Kumamoto, Japan) was used to assay PRF exudate for its effects on hPDLC proliferation. hPDLCs $\left(2 \times 10^{3} / 100 \mu \mathrm{l} /\right.$ well $)$ were seeded into each well of 96 -well plates containing $10 \%$ FBS complete medium and incubated for $24 \mathrm{~h}$. Next, $50 \mu \mathrm{l}$ 


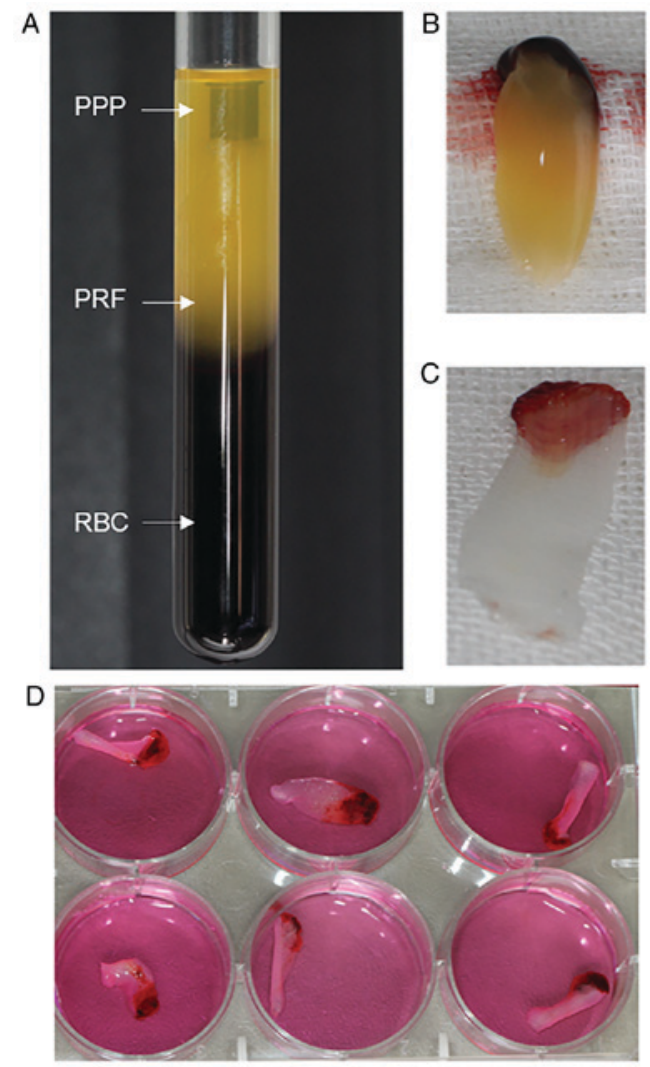

Figure 1. The production of PRF exudate. (A) Following centrifugation, the blood samples formed three layers. The upper layer contained PPP, the bottom layer was comprised of RBCs and the middle layer was the PRF clot (B) The PRF clot was separated from the upper part of the RBC layer. (C) The PRF clot was squeezed between two sterile gauzes to form a PRF membrane. (D) The PRF membrane was added to $\alpha$-minimum essential medium. PPP, platelet-poor plasma; RBCs, red blood cells; PRF, platelet-rich fibrin.

medium supplemented with 4,20 , or $100 \%$ concentration of PRF exudate were added to each well and incubated for 24 , 48 , or $72 \mathrm{~h}$. Cell proliferation was calculated using the CCK-8 at specified experimental time points. The kit reagent $(10 \mu \mathrm{l})$ was added to the culture medium of each well. Following a 90 min incubation, the absorbance at a wavelength of $450 \mathrm{~nm}$ was detected using an automatic microplate reader (Infinite 200 PRO; Tecan Group Ltd., Mannedorf, Switzerland). The well containing medium and CCK-8 solution minus seeded cells was used as the blank control. The assay was performed in duplicate and the trial repeated six times under the same conditions.

Assay of ALP activity. hPDLCs $(500 \mu \mathrm{l})$ were seeded into each well of 24-well plates (Corning Incorporated, Corning, NY, USA) at a density of $1 \times 10^{4} /$ well in $10 \%$ FBS complete medium and incubated for $24 \mathrm{~h}$. Next, the cells were exposed to PRF exudate at various concentrations [100\% (E1), 20\% (E2) or 4\% (E3)] at 7 or 14 days. At the given time points, the cells were lysed with $0.1 \%$ Triton $\mathrm{X}-100$ and the lysates were centrifuged at $8,000 \mathrm{x} \mathrm{g}$ for $10 \mathrm{~min}$ at $4^{\circ} \mathrm{C}$. Supernatant $(50 \mu \mathrm{l} /$ well $)$ was added to 96-well plates and the ALP activity was examined using the ALP assay kit (Nanjing Jiancheng Bioengineering Institute, Nanjing, China). The optical density values were read ata wavelength of $520 \mathrm{~nm}$ with an automatic microplate reader (Infinite200 PRO; Tecan Group Ltd.).
Osteogenic differentiation induction. hPDLCs $\left(1 \times 10^{4} / 500 \mu \mathrm{l} /\right.$ well $)$ were seeded into each well of 24 -well plates in normal medium until they reached $60-70 \%$ confluence. The medium was then replaced with four different media: Standard medium (10\% FBS) or standard medium supplemented with three different concentrations of PRF exudate: $100 \%$ (E1), $20 \%$ (E2), or $4 \%$ (E3). The cells in the three experimental groups with E1, E2 and E3 were all maintained in osteogenic differentiation medium [Dulbecco's Modified Eagle Medium (DMEM) supplemented with 10\% FBS, $50 \mathrm{nM}$ dexamethasone, $0.2 \mathrm{mM}$ ascorbic acid, $10 \mathrm{mM}$ b-glycerophosphate and 100 units/ml penicillin-streptomycin]. Each well in the 24-well plate contained a specific concentration as follows: DMEM only, E1, E2, or E3. In addition, the three experimental groups with E1, E2 and E3 also contained mineralization-induction medium ( $\alpha$-MEM containing $10 \% \mathrm{FBS}, 50 \mathrm{mg} / \mathrm{ml}$ ascorbic acid, $10 \mathrm{mmol} / 1 \beta$-glycerol phosphate and $10^{-8} \mathrm{M}$ dexamethasone). Only the control group containing DMEM alone did not contain mineralization-induction medium. The medium was changed every 3 days.

Alizarin red S staining. Formation of the mineralized nodules was detected and quantified following 14 days by an Alizarin red-based assay. This assay was carried out according to the previous protocol with minor modifications (18). Briefly, hPDLCs were fixed with $95 \%$ ethanol for $15 \mathrm{~min}$ at room temperature. The cells were then washed twice with $\mathrm{dH}_{2} \mathrm{O}$ and stained with $0.1 \%$ alizarin red $\mathrm{S}$ solution $(\mathrm{pH} 4.1$ ) for $20 \mathrm{~min}$ at room temperature. Next, the cells were washed three times with $\mathrm{dH}_{2} \mathrm{O}$. To semi-quantify the content of mineralized matrix nodules, $100 \mathrm{mM}$ cetyl pyridinium chloride was added to the 24-well plates to dissolve and release calcium-combined alizarin red $\mathrm{S}$ into solution. The optical density values were read at $570 \mathrm{~nm}$, which represented the relative quantity of mineralization nodules. A total of 14 days post-osteogenic induction, mineralized nodules were observed by inverted phase-contrast microscopy (IX73; magnification, x20; Olympus Corporation, Tokyo, Japan).

Western blotting. Cell lysates were prepared in radioimmunoprecipitation buffer (150 mM NaCl, 0.1\% SDS, $1 \mathrm{mM}$ PMSF, $10 \mathrm{mM}$ Tris- $\mathrm{Cl}, \mathrm{pH} 7.4,1 \%$ sodium deoxycholate and $1 \%$ Triton X-100). The cells were treated with four different media for 3,5 , or 7 days. The cell lysates were incubated for $30 \mathrm{~min}$ on ice, then clarified by centrifugation at $6,000 \mathrm{x} g$ for $10 \mathrm{~min}$ at $4^{\circ} \mathrm{C}$. The protein contents were quantified with a bicinchoninic acid assay. Protein samples $(20 \mu \mathrm{l})$ were denatured and resolved by $10 \%$ SDS-PAGE, transferred onto a polyvinylidene difluoride membrane (EMD Millipore), and run at $300 \mathrm{~mA}$ for $2 \mathrm{~h}$. The membranes were blocked by incubating with $5 \%$ non-fat milk at room temperature for $1 \mathrm{~h}$, then incubated with primary anti-RUNX2 antibody (1:1,000; cat. no. 12556; Cell Signaling Technology) overnight at $4^{\circ} \mathrm{C}$. The primary antibody was then removed by washing the membrane three times in Tris-buffered saline containing 0.1\% Tween 20 (TBST) and incubated with horseradish peroxidase-goat anti-rabbit immunoglobulin G (1:500; cat. no. 10285-1-AP; Proteintech Group) for $1 \mathrm{~h}$ at room temperature. Following three washes with TBST, the protein bands were visualized using the Enhanced Chemiluminescence kit (GE Healthcare, Chicago, IL, USA) 
and exposed to X-ray film. $\beta$-actin (1:1,000; cat. no. A5441; Sigma-Aldrich; Merck KGaA) was used as internal reference.

Reverse transcription-quantitative polymerase chain reaction $(R T-q P C R)$. hPDLCs $\left(1 \times 10^{5} / 2 \mathrm{ml} /\right.$ well $)$ were seeded into each well of 6-well plates in standard medium until they reached $60-70 \%$ confluence. The cells were treated with four different media. The control group was osteogenic-inducing medium [DMEM plus $50 \mu \mathrm{g} / \mathrm{ml}$ ascorbic acid and $10 \mathrm{mM} \beta$-sodium glycerophosphate (Sigma-Aldrich; Merck KGaA)]. The three experimental groups were treated with osteogenic-inducing medium plus a PRF exudate dilution (E1, E2 or E3). RNA was extracted using TRIzol ${ }^{\circledast}$ (Invitrogen; Thermo Fisher Scientific, Inc.) and reverse transcribed into cDNA on the 3rd, 5th and 7th days. cDNA synthesis was performed with $1 \mu \mathrm{g}$ total RNA using SuperScript II reverse transcriptase and random hexamer primers (cat. no. 18064014; Invitrogen; Thermo Fisher Scientific, Inc.). The temperature protocol used was as follows: Room temperature for $10 \mathrm{~min}$ and then $37^{\circ} \mathrm{C}$ for $60 \mathrm{~min}$; followed by $85^{\circ} \mathrm{C}$ for $5 \mathrm{~min}$.

An aliquot $(2 \mu \mathrm{l})$ of each sample was used for quantitative (q) PCR determination of the expression of the osteogenic genes $O C N, R U N X 2$ and $O S X$ using the $\mathrm{SYBR}^{\circledR}$ Premix Ex Taq $^{\text {TM }}$ II kit (Takara Bio, Inc., Otsu, Japan). qPCR primers were designed to span an intron so that only RNA-specific amplification was possible. PCR was performed in a Rotor-Gene thermocycler as follows: $95^{\circ} \mathrm{C}$ for $3 \mathrm{~min}, 40$ cycles of $95^{\circ} \mathrm{C}$ for $3 \mathrm{sec}$ and $60^{\circ} \mathrm{C}$ for $60 \mathrm{sec}$. Each sample was tested in triplicate and fold differences in gene expression were calculated using the $2^{-\Delta \Delta C q}$ method (19) with normalization to human $\beta$-actin. The primer design is presented in Table I.

Statistical analysis. Data are presented as the mean \pm standard deviation of 3-4 independent experiments, and statistical analyses were performed with SPSS 17.0 software (SPSS, Inc., Chicago, IL, USA). The cell proliferation and ALP activity assays were analyzed by one-way analysis of variance (ANOVA) and Tukey's multiple comparison tests The western blotting and Alizarin red staining assay data were analyzed using two-way ANOVA followed by Bonferroni's post-hoc comparisons test for independent samples. $\mathrm{P}<0.05$ was considered to indicate a statistically significant difference.

\section{Results}

Characterization of $h P D L C s$. hPDLCs grew from the explant following 7-10 days of culture, a spindle shape was observed and certain cells were distributed in a circinate alignment pattern with rapid proliferation (Fig. 1A and B). The PRF exudate obtained was a clear, yellowish fluid. Each 50-ml blood sample produced $4.5 \mathrm{ml}$ of PRF exudate. The cells were vimentin positive (Fig. 1C) and keratin negative (Fig. 1D), according to immunocytochemistry staining, which indicated that the primary cells were of mesenchymal origin.

Effects of PRF exudate on PDLC proliferation. The objective of the present study was to examine the impact of various concentrations of hPRF exudate on the proliferation of hPDLCs in vitro. The hPRF was obtained according to the previous protocol (Fig. 2) (8). The cells were exposed to varying
Table I. Primers used for reverse transcription-quantitative polymerase chain reaction analysis.

\begin{tabular}{llc}
\hline Gene & \multicolumn{1}{c}{ Primer sequences (5'-3') } & PCR bp \\
\hline$\beta$-actin & & \\
$\mathrm{F}$ & AGAAAATCTGGCACCACACC & 139 \\
$\mathrm{R}$ & GGGGTGTTGAAGGTCTAAA & \\
Osteocalcin & & \\
$\mathrm{F}$ & GGCGCTACCTGTATCAATGG & 106 \\
$\mathrm{R}$ & TCAGCCAACTCGTCACAGTC & \\
RUNX2 & & \\
$\mathrm{F}$ & CACCATGTCAGCAAAACTTCTT & 96 \\
$\mathrm{R}$ & TCACGTCGCTCATTTTGC & \\
Osterix & & \\
$\mathrm{F}$ & TGCTTGAGGAGGAAGTTCAC & 148 \\
$\mathrm{R}$ & AGGTCACTGCCCACAGAGTA & \\
\hline
\end{tabular}

RUNX2, runt-related transcription factor 1; PCR, polymerase chain reaction; F, forward; $\mathrm{R}$, reverse.

concentrations of PRF exudate for 24,48 , or $72 \mathrm{~h}$. The viability and proliferation of hPDLCs were determined by using a CCK-8 kit assay (Fig. 3). On day 1, the difference between the control and experimental groups was significant $(\mathrm{P}<0.05)$, while the difference between the E1 and E2 experimental groups was not significant $(\mathrm{P}>0.05)$, and the difference between $\mathrm{E} 1$ and $\mathrm{E} 3$ experimental groups was significant $(\mathrm{P}<0.01$; Fig. 3). On days 2 and 3 , when compared with the control group, the proliferation rate of groups E1 and E2 increased significantly $(\mathrm{P}<0.01)$. The PRF exudates in group E3 also enhanced the proliferation of hPDLCs, although there was no significant difference between group E3 and the control group ( $P>0.05)$. On days 2 and 3, the difference between groups E1 and E2 was significant $(\mathrm{P}<0.05)$ as was the difference between groups $\mathrm{E} 1$ and $\mathrm{E} 3(\mathrm{P}<0.05)$, while the difference between groups E2 and E3 was only significant at the day 2 time interval $(\mathrm{P}<0.01)$.

ALP activity. Following 7 or 14 days of culture, the hPDLCs cultured in the E3 group demonstrated the highest levels of ALP activity compared with the other experimental groups and the differences between the control group, and the E2 and E3 experimental groups were significant $(\mathrm{P}<0.01)$ at all time intervals compared with the control (Fig. 4). Furthermore, the ALP activity of hPDLCs in the E1 group on days 7 and 14 were also upregulated, although the difference was not significant $(\mathrm{P}>0.05)$. As expected, ALP activity in all groups progressively increased over time in culture.

Alizarin red staining and semi-quantification of mineralized matrix nodules. To detect the formation of mineralized matrix nodules, alizarin red staining was performed (Fig. 5A). Following 14 days of osteogenic induction, the mineralized nodules were observed by inverted phase-contrast microscopy and the number of nodules was increased in the MM + E1 group compared with the MM group. However, it was hard to detect mineralized nodules in the control group. 

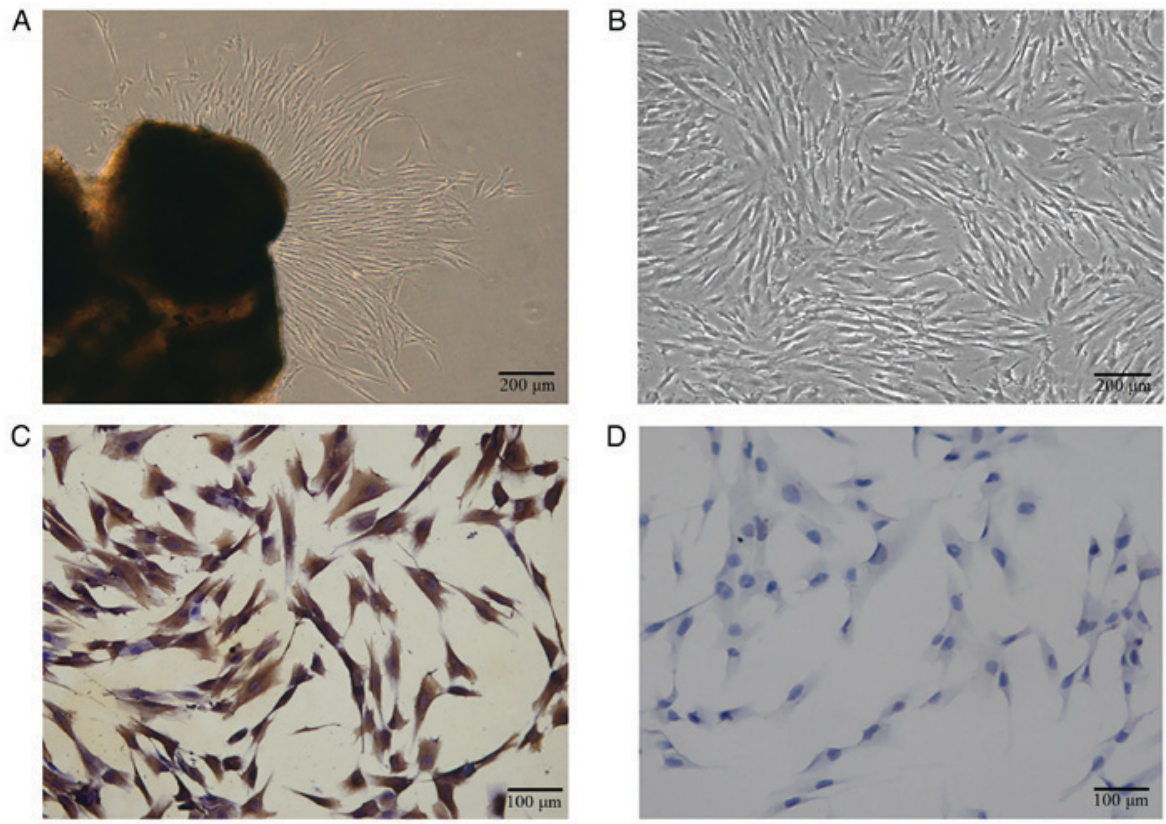

Figure 2. Characterization and identification of hPDLCs (A) Primary cells grew out from tissue explants. (B) The primary hPDLCs exhibited a spindle shape. (C) Immunocytochemistry staining was positive for vimentin. (D) Immunocytochemistry staining was negative for cytokeratin. For (A) and (B): Scale bar, $200 \mu \mathrm{m}$. For (C) and (D): Scale bar, $100 \mu \mathrm{m}$. hPDLCs, primary human periodontal ligament cells.

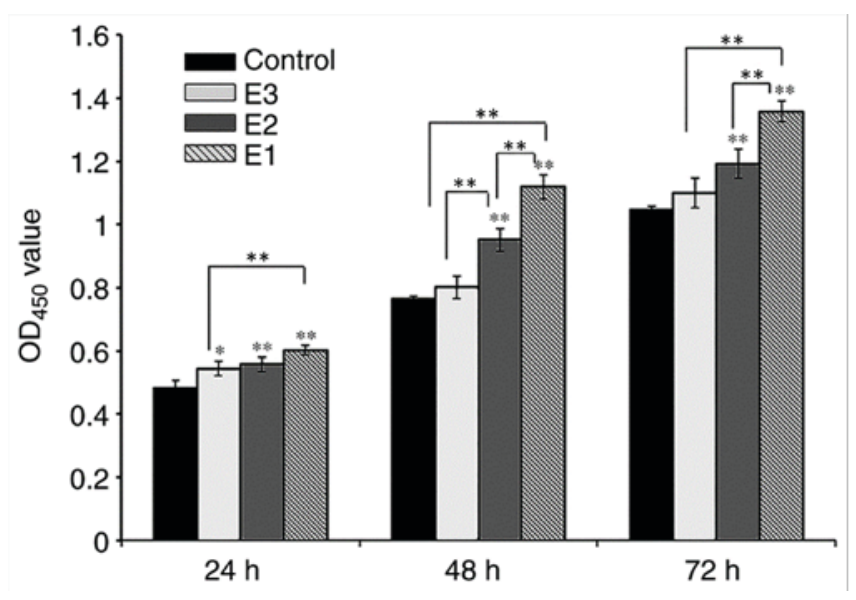

Figure 3. Effects of PRF exudate on the proliferation of hPDLCs in vitro. The proliferation activity of hPDLSCs was promoted by PRF exudates in a dose-dependent manner. E1, 100\%, E2, 20\%; E3, 4\% PRF exudate. "P<0.05 and ${ }^{* *} \mathrm{P}<0.01$. hPDLCs, primary human periodontal ligament cells; PRF, platelet-rich fibrin; OD, optical density.

Furthermore, the formation level of mineralized matrix nodules were quantified following 14 days of induction (Fig. 5B). The absorbance values at $570 \mathrm{~nm}$ revealed that extracellular calcium deposition in the E1 group and the $\mathrm{MM}+\mathrm{E} 1$ group was significantly increased compared with the control group or MM group, respectively $(\mathrm{P}<0.05)$.

Effect of PRF on the expression of osteogenic-associated genes. The results of the expression levels of $O C N, O S X$ (also known as SP7) and RUNX2 genes were recorded on the 3rd, 5 th, and 7 th days. It was observed that on the 3 rd day, the expression of osteogenic-associated genes RUNX2 and the later marker $O S X$, were all upregulated in the PRF treatment groups vs. the corresponding control and MM group, and the

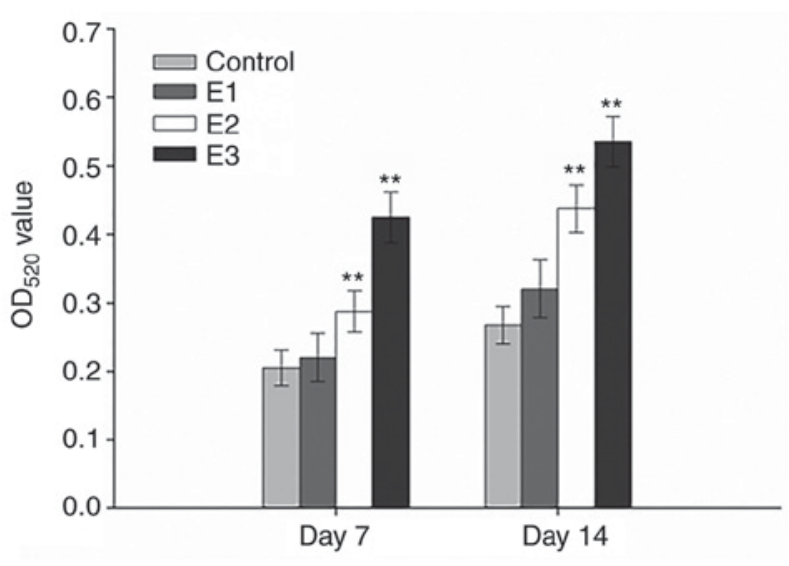

Figure 4. Effects of PRF exudate on ALP activity in human periodontal ligament cells. Following 7 days of culture, ALP activity E2 and E3 groups were significantly increased compared with the control group. Furthermore, compared with the control group, the ALP activity in the E1 group was also elevated, although the difference was not significant. ${ }^{* *} \mathrm{P}<0.01$ vs. the corresponding control. ALP, alkaline phosphatase; PRF, platelet-rich fibrin; OD, optical density.

differences became significant by the 5th day (Fig. 6A and B). Following the addition of the PRF exudate, the expression level of the other late marker, $O C N$, decreased on the 3rd day, but was upregulated in the experimental groups during the following period and was enhanced significantly by the 7 th day $(\mathrm{P}<0.01$; Fig. $6 \mathrm{C})$.

Western blotting was performed to examine the effect of PRF exudate on hPDLC differentiation and confirm the qPCR results. Cells were cultured in four varied media for 3, 5, or 7 days. As demonstrated in Fig. 7A and B, compared with the control group and the MM group, the protein levels of RUNX2 were upregulated in the E1-treated group and the $\mathrm{MM}+$ E1-treated group at different time points. 

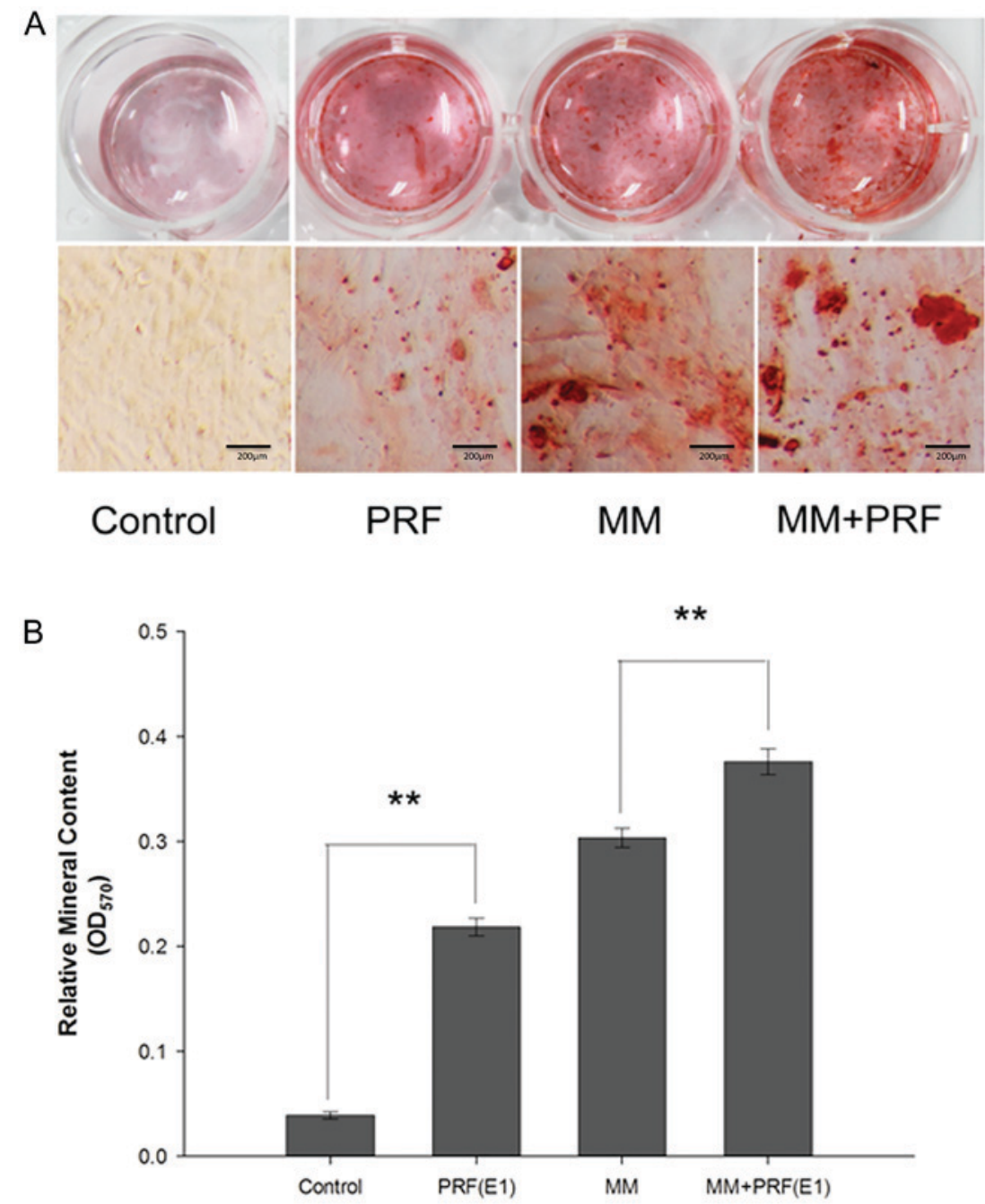

Figure 5. Alizarin red staining of periodontal ligament cells and semi-quantification of mineralized nodules. Four kinds of media: Control medium, E1, MM and $\mathrm{E} 1+\mathrm{MM}$. Scale bar, $200 \mu \mathrm{m}$. (A) Following 14 days of osteogenic induction, the number of mineralized nodules in the E1 + MM group was much greater than in the MM group. Furthermore, the E1 group exhibited an increased number of mineralized nodules compared with the control group. (B) The relative mineralized nodule content in the E1 + MM group was increased compared with the MM group. In addition, compared with the control group, the relative mineralized nodule content also increased. ${ }^{* *} \mathrm{P}<0.01$; PRF, platelet-rich fibrin; MM, mineralization-inducing medium; E1, 100\% PRF exudate medium.

\section{Discussion}

PRF has been described as a second-generation platelet concentrate, since it is produced naturally without using an anticoagulant. Platelet-rich plasma contains various growth factors, coagulation factors, adhesion molecules, cell factors, chemokines and binding elements (20). When the platelets are activated, the $\alpha$ granules release a large variety of growth factors (21) that promote the proliferation and differentiation of mesenchymal stem cells (22). The PRF clot forms an active fibrin matrix with a complex three-dimensional architecture, in which most of the platelets and leukocytes are trapped. Compared with platelet-rich plasma, PRF obtained by means of Choukroun's protocol does not decompose quickly following application; instead, the active PRF membrane is slowly remodeled in a similar way to a natural blood clot, and releases large amounts of factors and other matrix glycoproteins for at least 7 days.

The goal of the present study was to assess the in vitro effect of the soluble materials released from PRF membranes on the proliferation of hPDLCs. In this study, PRF demonstrated no cytotoxic impact on hPDLCs, according to the CCK-8 assay, which is considered to be more sensitive compared with assays based on tetrazolium salts, including MTT (23). Furthermore, the result is consistent with previous studies in other cell types $(24,25)$. Earlier studies proved that PRF stimulates proliferation of human bone mesenchymal stem cells, human osteoblast-derived osteosarcoma (SaOS2) cells, human keratinocyte-derived carcinoma (KB) cells and human fibroblast-derived lung carcinoma (MRC5) cells in a similar way $(26,27)$. However, there has been little study regarding the effects of PRF on hPDLCs until now. In the present study, the results of the CCK- 8 assay demonstrated that PRF promotes the proliferation of hPDLCs. The higher proliferation rate occurred when cells were treated with concentrations E1 (100\%) or E2 (20\%). For the lowest concentration, E3 (4\%), the improved effect on cell proliferation was weak or not significant. In other words, PRF stimulated hPDLC proliferation in a dose-dependent manner, even though there was not always a significant difference. 

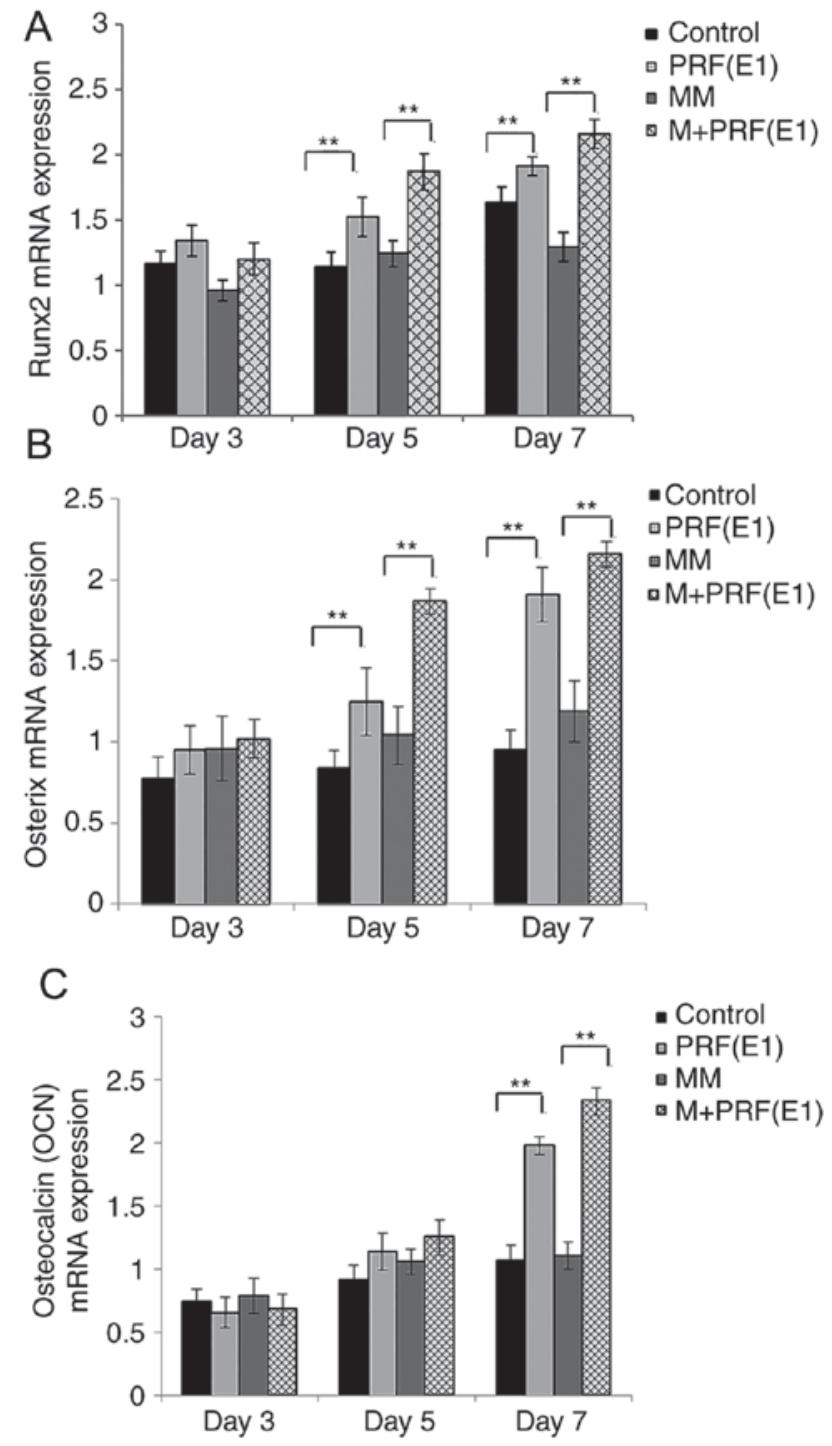

Figure 6. Reverse transcription-quantitative polymerase chain reaction results of three osteogenic-associated genes, RUNX2, OCN and $O S X$ (A) $R U N X 2$ and (B) $O S X$ were both upregulated in the PRF treatment groups vs. the corresponding controls. (C) $O C N$ expression was upregulated more slowly than RUNX2 and $O S X$ following exposure to PRF exudate. RUNX2, runt-related transcription factor 1; OCN, osteocalcin; OSX, osterix; PRF, platelet-rich; MM, mineralization-inducing medium. ${ }^{*} \mathrm{P}<0.05$ and ${ }^{* *} \mathrm{P}<0.01$ vs. corresponding control groups.

These data are consistent with the results reported in previous studies (28-30).

The enhanced effects on cell proliferation can be explained as follows. PRF releases platelet-derived growth factor (31), which is the primary growth factor in PRF and has been demonstrated to have potent mitogenic effects on stem cells, osteoblasts and dental pulp cells, and also promotes angiogenesis and collagen synthesis. Furthermore, other growth factors trapped in the PRF matrix (32), including insulin-like growth factor, vascular endothelial growth factor and basic fibroblast growth factor facilitate periodontal ligament stem cell and Schwann cell proliferation $(31,33)$, although these cells are fewer in number. Therefore, all of these growth factors together enhance the proliferation of hPDLCs.

In addition to the measurement of proliferation in the present study, the osteogenic differentiation of hPDLCs
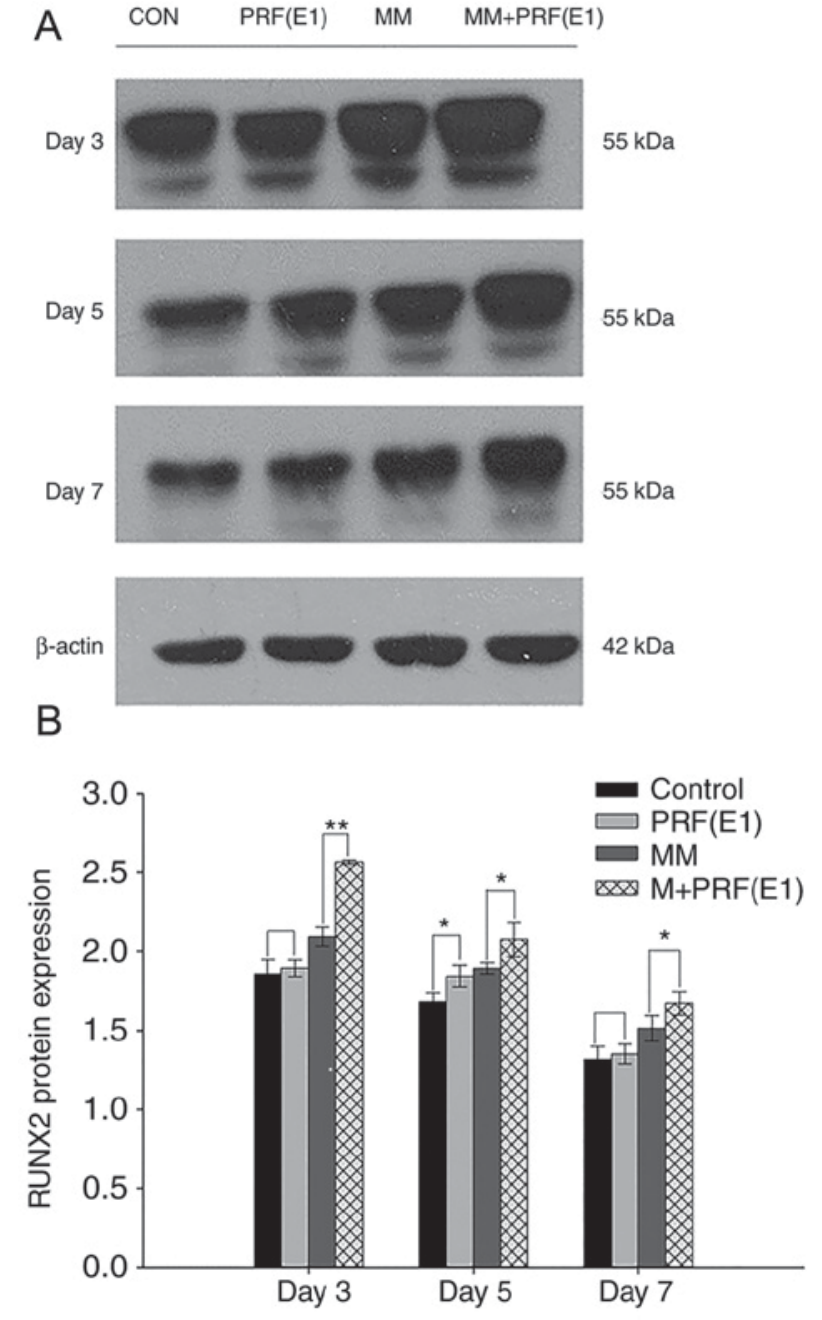

Figure 7. Protein expression of RUNX2 following treatment with various culture conditions for 3,5 , or 7 days. (A) Representative image and (B) semi-quantification of protein levels of RUNX2 detected via western blotting. $\beta$-actin was used to monitor equal protein loading. PRF, platelet-rich; MM, mineralization-inducing medium; CON, control. ${ }^{*} \mathrm{P}<0.05$ and ${ }^{* *} \mathrm{P}<0.01$ vs. corresponding control groups.

was also assessed by analyzing ALP activity, the formation of mineralized nodules and expression of the early-phase osteoblast-associated protein RUNX2.

ALP is a membrane enzyme that hydrolyzes phosphate ions and allows for the formation of hydroxyapatite crystals and the enhancement of mineralization (34). It is also regarded as a marker of osteoblastic differentiation at an early phase. Alizarin red is a marker that directly reflects the osteogenic differentiation of human umbilical-cord-derived mesenchymal stem cells (35). In this study, ALP activity was increased following stimulation with PRF. Similar effects have been reported in which PRF enhances ALP activity in osteoblasts, bone marrow mesenchymal stem cells and dentritic cell precursors (20). These results demonstrated that PRF contributes to the differentiation of hPDLSCs within a short period.

RUNX2 is a bone transcription factor and is one of the homologs of the Drosophila runt protein family. It was previously demonstrated that RUNX2 serves a significant role in osteogenesis and acts as an early transcriptional regulator in the process of osteogenic differentiation. Several studies have 
observed that $R U N X 2$ gene-deficient mice exhibit a complete block in bone formation and osteoblast differentiation $(36,37)$. Additionally, RUNX2 is essential for mesenchymal cell differentiation into an osteoblastic lineage (36). In accordance with the ALP result in the present study, the expression of RUNX2 was upregulated by PRF treatment. Based on the results that the expression of RUNX2 is increased following induction by PRF, it can be inferred that PRFs affects osteogenesis by promoting the differentiation of hPDLSCs into the osteogenic lineage.

Following analysis of this result, which growth factor contributes to the effect observed in the present study was investigated. RUNX2 is the primary binding protein induced by TGF- $\beta 1$ and bone morphogenetic protein 2 . In addition, TGF- $\beta$ is one of the major growth factors demonstrated in $\mathrm{PRF}$ and it is also involved in the regulation of multi-lineage differentiation of cells through the mothers against decapentaplegic homolog (SMAD) and non-SMAD pathways, including the mitogen-activated protein kinase, phosphoinositol-3 kinase-protein kinase B, and Rho-like GTPase signaling pathways (38-40). For example, TGF- $\beta$ promotes the differentiation of stem cells into cardiomyocytes, neurocytes, hepatic stellate cells, dendritic cells and other cell types (41).

In addition to the function of growth factors in PRF, it is worthwhile to investigate the role of the leukocytes that are released by PRF. To a certain extent, cell cultures that are treated with PRF membrane or exudate are a co-culture with leukocytes. However, most of the literature concerning platelet concentrates ignores the impact of these leukocytes, although a previous study has discussed the issue concerning the immune and antimicrobial properties of platelet concentrates (42).

PRF, due to its particular architecture, low expense and ease of production, may be a good choice for periodontal tissue regeneration. The promotion of the proliferation and differentiation of hPDLSCs also indicates the possibility of producing a PRF-hPDLSC conglomerate with several clots of PRF and a relatively small number of hPDLSCs. Although it has been demonstrated that PRF significantly facilitates hPDLSC proliferation and osteogenic differentiation, the ability to simulate clinical conditions may be limited, and the precise mechanism has yet to be proven. Finally, a limitation of the present study is that the blood samples and hPDLCs were not collected from the same donors, and the immune incompatibility may have an adverse effect on the experimental results.

In conclusion, we evaluated the effects of PRF exduate on the proliferation, osteogenic differentiation and mineralization of hPDLCs in vitro and the results showed that PRF exduate could enhance hPDLC adhesion, proliferation and induce the differentiation of hPDLC into mineralized tissue formation cell; thereby contribute to the main processes of periodontal tissue regeneration. PRF exduate may therefore provide potential benefits for periodontal tissue engineering; contributing to the primary processes of periodontal tissue regeneration. For economical and biological reasons, PRF exduate has greater clinical benefits than analogous growth factors. Furthermore, PRF exduate may be used to improve the early healing process of periodontal infrabony defects in clinical practice, as it may attenuate angiogenesis and prevent infectious activity. Future studies should investigate the underling mechanism of the association between PRF exduate and the promotion of osteogenic differentiation and mineralization of hPDLCs, as well as the effect of PRF exudate on the regeneration of soft and hard periodontal tissue following infection-induced inflammation.

\section{Acknowledgements}

The authors are grateful to the patients and their families for participating in the study.

\section{Funding}

This work was supported by grant from the People's Hospital of Longhua (Shenzhen, China) and the Health and Family Planning Commission of Shenzhen Municipality (grant no. SZFZ2018035).

\section{Availability of data and materials}

All data generated or analyzed during this study are included in this published article.

\section{Authors' contributions}

XL, HY and BW conceived of and designed the study. XL, YZ and HL performed cell culture, immunostaining and proliferation analysis. XL and YZ performed osteogenic differentiation induction, quantitative polymerase chain reaction and western blotting. $\mathrm{HY}, \mathrm{ZZ}$ and $\mathrm{ZY}$ provided reagents and performed data interpretation. XL, HY and BW performed data analysis and wrote the manuscript.

\section{Ethics approval and consent to participate}

The present study was approved by the Ethics Committee of the Jilin University Health Science Center (Jilin, China), and subjects provided informed consent.

\section{Patient consent for publication}

Patients gave their informed consent.

\section{Competing interests}

The authors declare that they have no competing interests.

\section{References}

1. Villar CC and Cochran DL: Regeneration of periodontal tissues: Guided tissue regeneration. Dent Clin North Am 54: 73-92, 2010.

2. Reynolds MA, Aichelmann-Reidy ME and Branch-Mays GL: Regeneration of periodontal tissue: Bone replacement grafts. Dent Clin North Am 54: 55-71, 2010.

3. Pietruska M, Pietruski J, Nagy K, Brecx M, Arweiler NB and Sculean A: Four-year results following treatment of intrabony periodontal defects with an enamel matrix derivative alone or combined with a biphasic calcium phosphate. Clin Oral Investig 16: 1191-1197, 2012.

4. Seo BM, Miura M, Gronthos S, Bartold PM, Batouli S, Brahim J, Young M, Robey PG, Wang CY and Shi S: Investigation of multipotent postnatal stem cells from human periodontal ligament. Lancet 364: 149-155, 2004.

5. Silvério KG, Rodrigues TL, Coletta RD, Benevides L, Da Silva JS Casati MZ, Sallum EA and Nociti FH Jr: Mesenchymal stem cell properties of periodontal ligament cells from deciduous and permanent teeth. J Periodontol 81: 1207-1215, 2010. 
6. Dohan DM, Choukroun J, Diss A, Dohan SL, Dohan AJ, Mouhyi J and Gogly B: Platelet-rich fibrin (PRF): A second-generation platelet concentrate. Part III: Leucocyte activation: A new feature for platelet concentrates. Oral Surg Oral Med Oral Pathol Oral Radiol Endod 101: e51-e55, 2006.

7. Choukroun J, Diss A, Simonpieri A, Girard MO, Schoeffler C, Dohan SL, Dohan AJ, Mouhyi J and Dohan DM: Platelet-rich fibrin (PRF): A second-generation platelet concentrate. Part IV: Clinical effects on tissue healing. Oral Surg Oral Med Oral Pathol Oral Radiol Endod 101: e56-e60, 2006.

8. Choukroun J, Diss A, Simonpieri A, Girard MO, Schoeffler C, Dohan SL, Dohan AJ, Mouhyi J and Dohan DM: Platelet-rich fibrin (PRF): A second-generation platelet concentrate. Part V: histologic evaluations of PRF effects on bone allograft maturation in sinus lift. Oral Surg Oral Med Oral Pathol Oral Radiol Endod 101: 299-303, 2006.

9. Diss A, Dohan DM, Mouhyi J and Mahler P: Osteotome sinus floor elevation using Choukroun's platelet-rich fibrin as grafting material: A 1-year prospective pilot study with microthreaded implants. Oral Surg Oral Med Oral Pathol Oral Radiol Endod 105: 572-579, 2008.

10. Park SI, Lee HR, Kim S, Ahn MW and Do SH: Time-sequential modulation inexpression of growth factors from platelet-rich plasma (PRP) on the chondrocyte cultures. Mol Cell Biochem 361: 9-17, 2012

11. Albanese A, Licata ME, Polizzi B and Campisi G: Platelet-rich plasma (PRP) in dental and oral surgery: From the wound healing to bone regeneration. Immun Ageing 10: 23, 2013.

12. Lee JW, Kwon OH, Kim TK, Cho YK, Choi KY, Chung HY, Cho BC, Yang JD and Shin JH: Platelet-rich plasma: Quantitative assessment of growth factor levels and comparative analysis of activated and inactivated groups. Arch Plast Surg 40: 530-535, 2013

13. Simonpieri A, Del Corso M, Sammartino G and Dohan Ehrenfest DM: The relevance of Choukroun's platelet-Rich fibrin and metronidazole during complex maxillary rehabilitations using bone allograft. Part 1: A new grafting protocol. Implant Dent 18: 102-111, 2009.

14. Dohan Ehrenfest DM, de Peppo GM, Doglioli P and Sammartino G: Slow release of growth factors and thrombospondin-1 in Choukroun's platelet-rich fibrin (PRF): A gold standard to achieve for all surgical platelet concentrates technologies. Growth Factors 27: 63-69, 2009.

15. Dohan Ehrenfest DM, Diss A, Odin G, Doglioli P, Hippolyte MP and Charrier JB: In vitro effects of Choukroun's PRF (platelet-rich fibrin) on human gingival fibroblasts, dermal prekeratinocytes, preadipocytes, and maxillofacial osteoblasts in primary cultures. Oral Surg Oral Med Oral Pathol Oral Radiol Endod 108: 341-352, 2009.

16. He L, Lin Y, Hu X, Zhang Y and Wu H: A comparative study of platelet-rich fibrin (PRF) and platelet-rich plasma (PRP) on the effect of proliferation and differentiation of rat osteoblasts in vitro. Oral Surg Oral Med Oral Pathol Oral Radiol Endod 108 707-713, 2009

17. Tavakolinejad S, Khosravi M, Mashkani B Ebrahimzadeh-Bideskan A, Sanjar-Mossavi N, Parizadeh MR and Hamidi-Alamdari D: The effect of human platelet-rich plasma on adipose-derived stem cell proliferation and osteogenic differentiation. Iran Biomed J 18: 151-157, 2014.

18. Yu J, Deng Z, Shi J, Zhai H, Nie X, Zhuang H, Li Y and Jin Y: Differentiation of dental pulp stem cells into regular-shaped dentin-pulp complex induced by tooth germ cell conditioned medium. Tissue Eng 12: 3097-3105, 2006.

19. Livak KJ and Schmittgen TD: Analysis of relative gene expression data using real-time quantitative PCR and the 2(-Delta Delta C(T)) method. Methods 25: 402-408, 2001

20. Gassling V, Hedderich J, Acil Y, Purcz N, Wiltfang J and Douglas T: Comparison of platelet rich fibrin and collagen as osteoblast-seeded scaffolds for bone tissue engineering applications. Clin Oral Implants Res 24: 320-328, 2013.

21. Xie XT, Zhang CQ and Tuan RS: Biology of platelet-rich plasma and its clinical application in cartilage repair. Arthritis Res Ther 16: 204-211, 2014.

22. Jia X, Pan J, Li X, Li N, Han Y, Feng X and Cui J: Bone marrow mesenchymal stromal cells ameliorate angiogenesis and renal damage via promoting PI3k-Akt signaling pathway activation in vivo. Cytotherapy 18: 838-845, 2016.

23. González-Fernández ML, Pérez-Castrillo S, Sánchez-Lázaro JA, Prieto-Fernández JG, López-González ME, Lobato-Pérez S, Colaço BJ, Olivera ER and Villar-Suárez V: Assessment of regeneration in meniscal lesions by use of mesenchymal stem cells derived from equine bone marrow and adipose tissue. Am J Vet Res 77: 779-788, 2016.
24. An S, Ling J, Gao Y and Xiao Y: Effects of varied ionic calcium and phosphate on the proliferation, osteogenic differentiation and mineralization of human periodontal ligament cells in vitro. J Periodontal Res 47: 374-382, 2012

25. Huang FM, Yang SF, Zhao JH and Chang YC: Platelet-rich fibrin increases proliferation and differentiation of human dental pulp cells. J Endod 36: 1628-1632, 2010.

26. Chang IC, Tsai $\mathrm{CH}$ and Chang YC: Platelet-rich fibrin modulates the expression of extracellular signal-regulated protein kinase and osteoprotegerin in human osteoblasts. J Biomed Mater Res A 95: 327-332, 2010.

27. Clipet F, Tricot S, Alno N, Massot M, Solhi H, Cathelineau G, Perez F, De Mello G and Pellen-Mussi P: In vitro effects of Choukroun's platelet-rich fibrin conditioned medium on 3 different cell lines implicated in dental implantology. Implant Dent 21: 51-56, 2012

28. Han J, Meng HX, Tang JM, Li SL, Tang Y and Chen ZB: The effect of different platelet-rich plasma concentrations on proliferation and differentiation of human periodontal ligament cells in vitro. Cell Prolif 40: 241-252, 2007.

29. Xia L, Zhang Z, Chen L, Zhang W, Zeng D, Zhang X, Chang J and Jiang X: Proliferation and osteogenic differentiation of human periodontal ligament cells on akermanite and $\beta$-TCP bioceramics. Eur Cell Mater 22: 68-83, 2011.

30. Zhao YH, Zhang M, Liu NX, Lv X, Zhang J, Chen FM and Chen YJ: The combined use of cell sheet fragments of periodontal ligament stem cells and platelet-rich fibrin granules for avulsed tooth reimplantation. Biomaterials 34: 5506-5520, 2013.

31. Dohan Ehrenfest DM, Doglioli P, de Peppo GM, Del Corso M and Charrier JB: Choukroun's platelet-rich fibrin (PRF) stimulates in vitro proliferation and differentiation of human oral bone mesenchymal stem cell in a dose-dependent way. Arch Oral Biol 55: 185-194, 2010.

32. Wang X, Zhang Y, Choukroun J, Ghanaati S and Miron RJ: Effects of an injectable platelet-rich fibrin on osteoblast behavior and bone tissue formation in comparison to platelet-rich plasma. Platelets 29: 48-55, 2018.

33. Yu Y, Mu J, Fan Z, Lei G, Yan M, Wang S, Tang C, Wang Z, $\mathrm{Yu} \mathrm{J}$ and Zhang G: Insulin-like growth factor 1 enhances the proliferation and osteogenic differentiation of human periodontal ligament stem cells via ERK and JNK MAPK pathways. Histochem Cell Biol 137: 513-525, 2012

34. Xu FT, Li HM, Yin QS, Liang ZJ, Huang MH, Chi GY, Huang L, Liu DL and Nan H: Effect of activated autologousplatelet-rich plasma on proliferation and osteogenicdifferentiation of human adipose-derived stem cells in vitro. Am J Transl Res 7: 257-270, 2015

35. Pereira Lopes FR, Lisboa BC, Frattini F, Almeida FM, Tomaz MA, Matsumoto PK, Langone F, Lora S, Melo PA, Borojevic R, et al: Enhancement of sciatic nerve regeneration after vascular endothelial growth factor (VEGF) gene therapy. Neuropathol Appl Neurobiol 37: 600-612, 2011.

36. Roohani-Esfahani SI, No YJ, Lu Z, Ng PY, Chen Y, Shi J, Pavlos NJ and Zreiqat H: A bioceramic with enhanced osteogenic properties to regulate the function of osteoblastic and osteocalastic cells for bone tissue regeneration. Biomed Mater 11: 35-38, 2016.

37. Fowlkes JL, Bunn RC, Liu L, Wahl EC, Coleman HN, Cockrell GE, Perrien DS, Lumpkin CK Jr and Thrailkill KM: Runt-related transcription factor 2 (RUNX2) and RUNX2-related osteogenic genes are down-regulated throughout osteogenesis in type 1 diabetes mellitus. Endocrinology 149: 1697-1704, 2008.

38. Marie PJ: Transcription factors controlling osteoblastogenesis. Arch Biochem Biophys 473: 98-105, 2008.

39. Chen L, Zou X, Zhang RX, Pi CJ, Wu N, Yin LJ and Deng ZL: IGF1 potentiates BMP9-induced osteogenic differentiation in mesenchymal stem cells through the enhancement of BMP/Smad signaling. BMB Rep 49: 122-127, 2016.

40. Sağsöz H, Liman N and Alan E: Expression of vascular endothelial growth factor receptors and their ligands in rat uterus during the postpartum involution period. Biotech Histochem 90: 361-374, 2015.

41. Corrado A, Neve A and Cantatore FP: Expression of vascular endothelial growth factor in normal, osteoarthritic and osteoporotic osteoblasts. Clin Exp Med 13: 81-84, 2013.

42. Wang MK, Sun HQ, Xiang YC, Jiang F, Su YP and Zou ZM Different roles of TGF- $\beta$ in the multi-lineage differentiation of stem cells. World J Stem Cells 4: 28-34, 2012. 